\title{
Détermination des écoulements préférentiels en zone karstique (Tafrata, Maroc), Apport des données satellitaires SAR ERS-1 et Landsat ETM+ et de la prospection géophysique Determination of the preferential flows in a karstic area (Tafrata, Morocco): Contribution of satellite SAR ERS-1 and Landsat ETM+ data and of the geophysical prospection.
}

\author{
Said Boutaleb, Fatima El Hammichi, Hassan Tabyaoui, Lhoussaine Bouchaou \\ et Khalid Dindane
}

Volume 22, numéro 3, 2009

URI : https://id.erudit.org/iderudit/037779ar

DOI : https://doi.org/10.7202/037779ar

Aller au sommaire du numéro

Éditeur(s)

Université du Québec - INRS-Eau, Terre et Environnement (INRS-ETE)

ISSN

1718-8598 (numérique)

Découvrir la revue

Citer cet article

Boutaleb, S., Hammichi, F. E., Tabyaoui, H., Bouchaou, L. \& Dindane, K. (2009). Détermination des écoulements préférentiels en zone karstique (Tafrata, Maroc), Apport des données satellitaires SAR ERS-1 et Landsat ETM+ et de la prospection géophysique. Revue des sciences de l'eau / Journal of Water Science, 22(3), 407-419. https://doi.org/10.7202/037779ar
Résumé de l'article

Malgré le grand nombre de puits et forages d'eau réalisés dans les différentes régions karstiques du Maroc, de nombreux inconnus subsistent encore concernant les critères d'implantation et le coût final des ouvrages. Afin d'optimiser les campagnes de forage à venir, une méthodologie de prospection multicritère a été adoptée dans la région karstique nord-orientale du Maroc. Elle s'articule selon trois axes, à savoir : 1) détermination des linéaments hydrogéologiques à partir du traitement et interprétation des images satellitaires radar SAR ERS-1 et Landsat ETM+;2) application de la géophysique électrique par (a) sondages électriques pour la détermination des épaisseurs des niveaux d'altération et de fracturation et par (b) trainées électriques pour la localisation précise de la zone de passage des linéaments hydrogéologiques, et finalement 3) application des techniques de Sondage par Résonance Magnétique (SRM) pour la vérification de la présence d'eau. Les différents résultats de sondages mécaniques réalisés à la suite de cette campagne de prospection montrent un taux de réussite très satisfaisant. 


\title{
DÉTERMINATION DES ÉCOULEMENTS PRÉFÉRENTIELS EN ZONE KARSTIQUE (TAFRATA, MAROC), APPORT DES DONNÉES SATELLITAIRES SAR ERS-1 ET LANDSAT ETM+ ET DE LA PROSPECTION GÉOPHYSIQUE
}

\author{
Determination of the preferential flows in a karstic area (Tafrata, Morocco): Contribution of \\ satellite SAR ERS-1 and Landsat ETM+ data and of the geophysical prospection.
}

Said Boutaleb ${ }^{1}$, Fatima El Hammichi ${ }^{2}$, Hassan Tabyaoui ${ }^{1 *}$, Lhoussaine Bouchaou ${ }^{3}$ Et Khalid Dindane $^{4}$

${ }^{1}$ Université Sidi Mohamed Ben Abdallah, Faculté Polydisciplinaire de Taza, B.P. 1223, Taza-Gare, Maroc

${ }^{2}$ Université Mohamed V, Faculté des Sciences, Département des Sciences de la Terre, B.P. 1014, Rabat-Agdal, Maroc

${ }^{3}$ Université Ibnou Zohr, Faculté des Sciences, Agadir, Maroc

${ }^{4}$ Bureau d'étude Anzar Conseil, Rabat, Maroc

\section{RÉSUMÉ}

Malgré le grand nombre de puits et forages d'eau réalisés dans les différentes régions karstiques du Maroc, de nombreux inconnus subsistent encore concernant les critères d'implantation et le coût final des ouvrages. Afin d'optimiser les campagnes de forage à venir, une méthodologie de prospection multicritère a été adoptée dans la région karstique nord-orientale du Maroc. Elle s'articule selon trois axes, à savoir: 1) détermination des linéaments hydrogéologiques à partir du traitement et interprétation des images satellitaires radar SAR ERS-1 et Landsat ETM+; 2) application de la géophysique électrique par (a) sondages électriques pour la détermination des épaisseurs des niveaux d'altération et de fracturation et par (b) trainées électriques pour la localisation précise de la zone de passage des linéaments hydrogéologiques, et finalement 3) application des techniques de Sondage par Résonance Magnétique (SRM) pour la vérification de la présence d'eau. Les différents résultats de sondages mécaniques réalisés à la suite de cette campagne de prospection montrent un taux de réussite très satisfaisant.
Mots clés: Écoulement karstique, images satellitaires, prospection géophysique, Tafrata, Maroc.

\begin{abstract}
In spite of the great number of water wells and drillings carried out in various karstic areas of Morocco, many unknown factors still remain concerning criteria for installing such works and for the final costs. To optimize future drilling campaigns, a multi-criteria method of prospecting was adopted in the karstic area of northeastern Morocco. It is presented according to three axes: 1) the determination of hydrogeologic lineaments based on the analysis and interpretation of SAR ERS- 1 and Landsat ETM+ images; 2) the application of electrical resistivity methods by (a) vertical electric soundings (VES) for the determination of the thicknesses of weathered layers and of fractures, and by (b) electric resistivity profiling for the
\end{abstract}


determination of the exact location of hydrogeological lineament zones; and finally, 3) the application of Magnetic Resonance Sounding (MRS) for the verification of the presence of water. The various results of mechanical drilling carried out following this prospecting campaign show a very satisfactory rate of success.

\section{Keys words: karstic flow, satellites images, geophysical pros- pecting, Tafrata, Morocco.}

\section{INTRODUCTION}

Satisfaire d'une manière urgente et immédiate les besoins en eau des populations, surtout des régions karstiques à déficit hydrique, conduit à une précipitation dans la réalisation des forages. Ce mode d'action justifie de nombreuses insuffisances: tâtonnements fréquents et pertes énormes de temps, d'argent et de matériel. La présente note a pour but l'élaboration d'une méthodologie d'étude qui permet de cibler des sites répondant aux deux critères suivants:

- meilleur potentiel aquifère souterrain localisé à des profondeurs accessibles et à des coûts compatibles avec les budgets attribués ;

recherche d'un nombre suffisant de cibles pour sécuriser les investissements et la gestion durable de la ressource à long terme.

Cette réponse prend la forme d'une étude combinée entre un outil cartographique d'aide à la décision (télédétection spatiale) accompagné d'une prospection géophysique (prospection électrique et Sondage par Résonance Magnétique (SRM)). Le traitement et l'interprétation des images satellitaires radar SAR ERS-1 et Landsat ETM+ permettront de détecter, en surface, les linéaments hydrogéologiques pouvant drainer les eaux infiltrées. La prospection géophysique permettra de préciser, d'une part, les caractéristiques des linéaments en profondeur par sondage et trainées électriques, et d'autre part, l'aptitude de drainage de ce linéament par le SRM. Cette démarche permet d'apporter des précisions pertinentes sur la présence de ressources exploitables dans les 100 premiers mètres de profondeur.

\section{CONTEXTE GÉOLOGIQUE}

La plaine de Tafrata correspond au domaine oriental, dont la subsidence est moindre du bassin néogène de Guercif. Ce dernier correspond à une vaste cuvette que comblent les dépôts néogènes et quaternaires. Il est situé au carrefour de quatre grandes unités morphologiques et structurales: les nappes pré-rifaines et les monts d'Oujda au nord, la chaîne intracontinentale du Moyen-Atlas et les Hauts-Plateaux au sud (Figure 1). La plaine de Tafrata est limitée à l'est par les Hauts-Plateaux. Ces derniers montrent à l'affleurement un socle paléozoïque constitué par de puissantes formations schisteuses, parfois métamorphisées, à quelques passées de grès arkosiques et de calcaires fossilifères d'âge Ordovicien à Carbonifère (BENZAQUEN, 1965). Ces dépôts, fortement fracturés, ont subi d'intenses phénomènes d'érosion. Ils sont surmontés par une série triasique, essentiellement continentale, constituée de couches rouges conglomératiques à éléments de socle, des coulées basaltiques inférieures, une intercalation d'un niveau carbonaté marin, des coulées basaltiques supérieures et au sommet, des couches rouges supérieures à pélites et conglomérats. Le Jurassique comprend des dolomies à la base, des calcaires et marno-calcaires du Lias et se termine par une dalle carbonatée du Dogger.

La plaine de Tafrata est limitée à l'ouest par des reliefs jurassiques de Haloua-Richa (Figure 1). Ces reliefs sont constitués de séries calcaréo-dolomitiques, structurées en plis déversés vers le SE par la tectonique alpine (COLETTA, 1977). Au centre, les dépôts jurassiques sont couverts par de faibles séquences marines et des dépôts continentaux néogènes à quaternaires (BERNINI et al., 1994; WERNLI, 1988). Ces dépôts montrent des variations d'épaisseur souvent liées à des subsidences différentielles en relation avec le jeu de quatre familles d'accidents: NE-SO, NO-SE, N-S et E-O. Les directions NE-SO et E-O sont des reviviscences tartihercyniennes. Les dépôts ont guidé l'évolution tectonosédimentaire mésozoïque et néogène à quaternaire. Ces failles sont responsables d'une tectonique en blocs basculés vers l'ouest et ont entretenu des subsidences différentielles plus ou moins importantes. Elles sont observables à l'affleurement dans les séries jurassiques des Hauts-Plateaux et du Moyen-Atlas et en subsurface dans le bassin de Guercif (ZIZI, 1996).

La présence d'aquifères potentiels dans les formations jurassiques de la plaine de Tafrata est fort probable et peut s'expliquer par le piégeage d'eau dans les faciès réservoirs calcaréo-dolomitiques. Les failles peuvent guider les écoulements préférentiels et alimenter les blocs adjacents par connexion latérale ou verticale. Les travaux de terrain montrent la présence d'une fracturation intense et complexe qu'il convient de ne pas négliger. Il s'agit de: 1) grandes fractures ouvertes, partiellement colmatées par des produits d'érosion marneux en surface; 2 ) failles normales mettant en contact des bancs calcaires sublithographiques avec des dolomies karstiques et 3) fractures orthogonales ouvertes puis entièrement colmatées par des produits de précipitation riches en calcaire. Ces failles jouent un rôle important dans le drainage des eaux, probablement à partir de l'ouest (Hauts-Plateaux calcaires) vers l'est. Le traitement numérique et l'interprétation des images satellitaires pourraient préciser les positions exactes des failles majeures en vue d'une analyse hydrogéologique. 


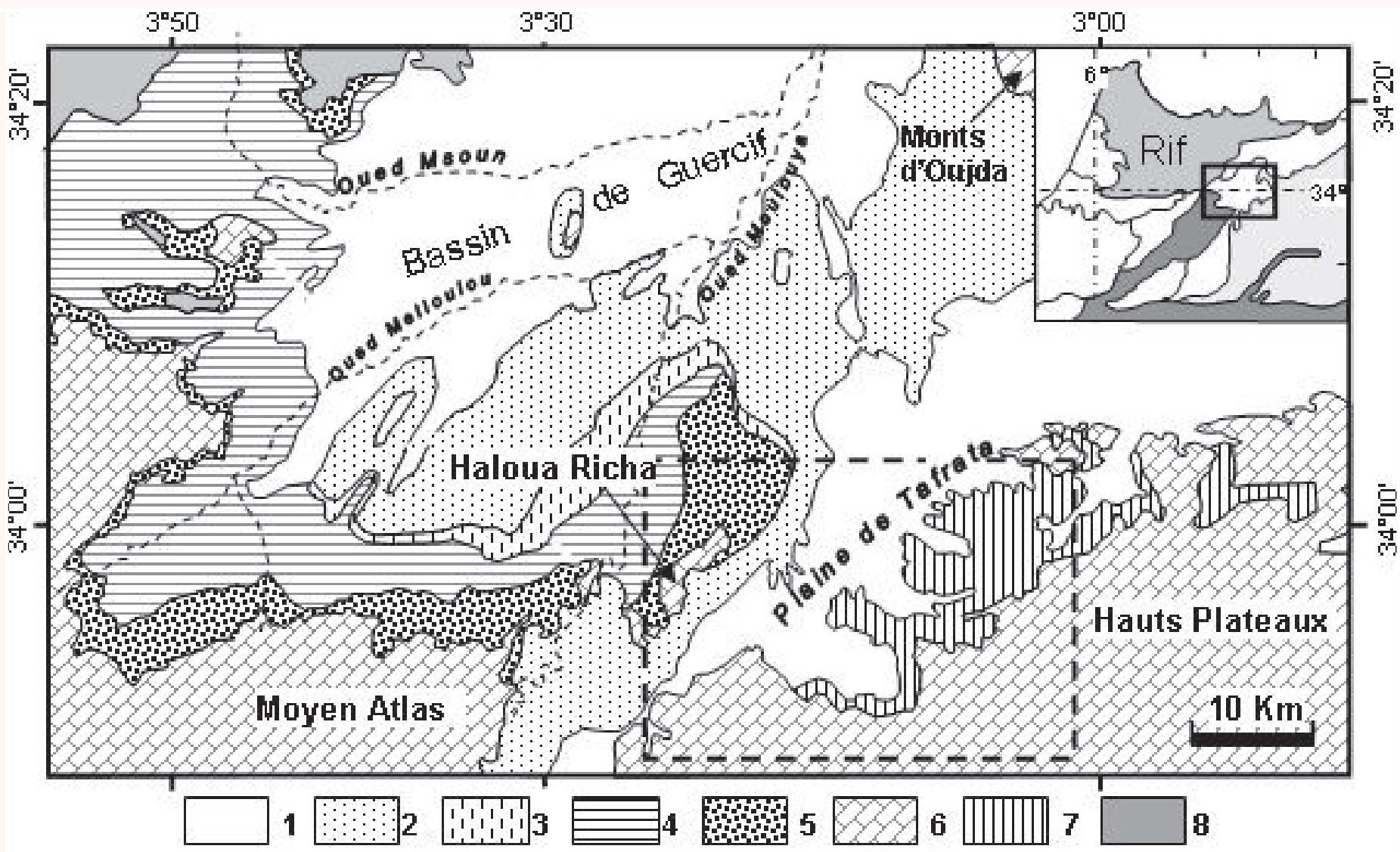

Figure 1. (a) Localisation du secteur d'étude au sein des domaines structuraux du nord du Maroc. (b) carte géologique simplifiée du basin de Guercif : (1) sédiments quaternaires, (2) formation de Bou Irhardaiene du Pliocène, (3) formation de Kef Ed Deba du Messinien, (4) formation Melloulou du Messinien-inférieur-Tortonien, (5) formation de Ras el Ksar et de Draa Sidi Saada d'âge Tortonien, (6) substratum mésozoïque, (7) socle hercynien et (8) la chaîne du Rif. Le cadre correspond au secteur de Tafrata.

(a) Location of the studied area within the main structural domains of northern Morocco. (b) Simplified Geological map of the Guercif Basin: (1) Quaternary sediments, (2) Pliocene, Bou Irbardaiene Fm., (3) Messinian, Kef ed Deba Fm., (4) Lower Messinian-Tortonian Melloulou Unit, (5) Tortonian Ras el Ksar and Draa Sidi Sada Fms, (6) Mesozoic substratum, (7) Hercynian metamorphic basement including Hercynian granites and (8) Rif Units.

\section{CARTOGRAPHIE STRUCTURALE PAR TÉLÉDÉTECTION ET SYSTÈME D'INFORMATION GÉOGRAPHIQUE}

\subsection{Données utilisées}

La base de données géoscientifiques comporte une image satellitaire ETM+ (scène 199-37 acquise le 04/03/2001) du satellite Landsat-7, une image radar SAR du satellite ERS-1 (scène 10903-2907, acquise le 16-08-1993) et une carte géologique au 1/ 500 000. Le traitement numérique des images a été réalisé à l'aide du logiciel ERSAS Imagin.

\subsection{Analyse et interprétation des images Landsat ETM+}

Le traitement numérique des images pour l'identification et l'extraction des linéaments est fondamental en matière de cartographie géologique et structurale (CONDID et CHAVEZ, 1979; SIEGAL et ABRAMS, 1976). Les exemples de travaux réalisés au Maroc, dans l'Anti-Atlas (EMRAN et al., 1988; HIMYARI et al., 2002; MAHMOUD 1996), la chaîne rifaine (CHOTIN et al., 1995) et les Béni Snassen (AIT BRAHIM et al., 2000), dans le bassin de Paris en France (ROUZEAU et SCANVIC, 1989), dans le Bouclier canadien (DESJARDINS et al., ; 2000 SHARMA et al., 1999) et dans bien d'autres régions, montrent que l'application des procédés de filtrage (spatiaux et directionnels) facilite l'accentuation des discontinuités lithologiques et structurales assimilables aux linéaments. Dans cette étude, le terme «linéament", défini par HOBBS (1904) et O'LEARY et al. (1976), décrit toute structure ayant un tracé rectiligne ou curviligne et dont l'interprétation a une signification géologique.

Les images ETM+ utilisées dans le présent travail sont acquises sans couverture nuageuse. Elles sont bien lisibles 
et s'adaptent aux analyses géologiques et structurales. Ces images ont fait l'objet de correction radiométrique et d'une rectification géométrique. Pour l'accentuation et la détection des linéaments, des filtrages directionnels ont été appliqués à diverses images. Les filtres de type haute fréquence, tels que le Laplacien, permettent de souligner les zones de variation brusque d'intensité. L'expérimentation avec des filtres Sobel de taille $5 \times 5,9 \times 9,15 \times 15$ et $31 \times 31$, et contenant des coefficients variés, a permis de conclure que le filtre $5 \times 5$ est le plus valable. Les filtres de grande taille créent des artefacts. Afin de maximiser l'extraction des linéaments dans toutes les directions, des filtres Sobel orientés à $0^{\circ}, 45^{\circ}, 90^{\circ}$ et $135^{\circ}$ sont appliqués sur l'image.

En appliquant les filtres de type Sobel à la seconde composante issue de l'analyse par composante principale (ACP), les principaux accidents ont été détectés. Dans les travaux de HIMYARI et al. (2002), l'application des filtres sur des images issues du traitement des ACP augmente de $20 \%$ l'ensemble des linéaments répertoriés. Des opérations arithmétiques (addition, soustraction, multiplication, division) sont faites pour combiner et transformer les images originales et/ou filtrées en de "nouvelles» images qui montrent plus clairement certaines structures.

Pour mieux faire ressortir les éléments structuraux (failles, joints, contacts lithologiques, dykes, etc.), la composition colorée en rouge-vert-bleu (RVB) des bandes 7, 4 et 2 a été utilisée. Le tracé des accidents coïncide souvent avec les cours d'eau qui sont soulignés par une végétation plus ou moins dense (Figure 2). La distinction des structures est également améliorée sur les trois premières composantes de l'ACP. On y distingue très bien le réseau hydrographique et les sols nus. Le relevé des linéaments est effectué par interprétation visuelle des images traitées: images brutes rehaussées, images transformées, compositions colorées. Les tracés rectilignes relatifs aux activités anthropiques (routes, pistes, lignes de transport d'énergie) sont éliminés de la carte obtenue par un croisement aux cartes topographiques existantes. Pour que la carte finale ne comporte que des linéaments géologiques, il est indispensable de procéder à une validation des linéaments. Les investigations géologiques, réalisées sur le terrain, ont permis de compléter et de valider la carte de fracturation établie (Figure 2). Cette carte constitue le support utilisé pour la simulation des écoulements souterrains de la plaine de Tafrata.

\subsubsection{Carte de fracturation}

Sur la carte obtenue (Figure 2), on distingue quatre directions tectoniques NE-SO, NO-SE, N-S et E-O :

- Les accidents NE-SO sont bien représentés au centre et à l'ouest du bassin de Tafrata. Ces accidents sont d'extension kilométrique et constituent la terminaison septentrionale des accidents du Moyen-Atlas. Le dernier jeu de ces failles est de type décrochant senestre et date de la fin du Miocène (AIT BRAHIM, 1986).

- Les failles de direction de NO-SE et N-S ont une répartition statistique prépondérante. Ces directions cartographiques

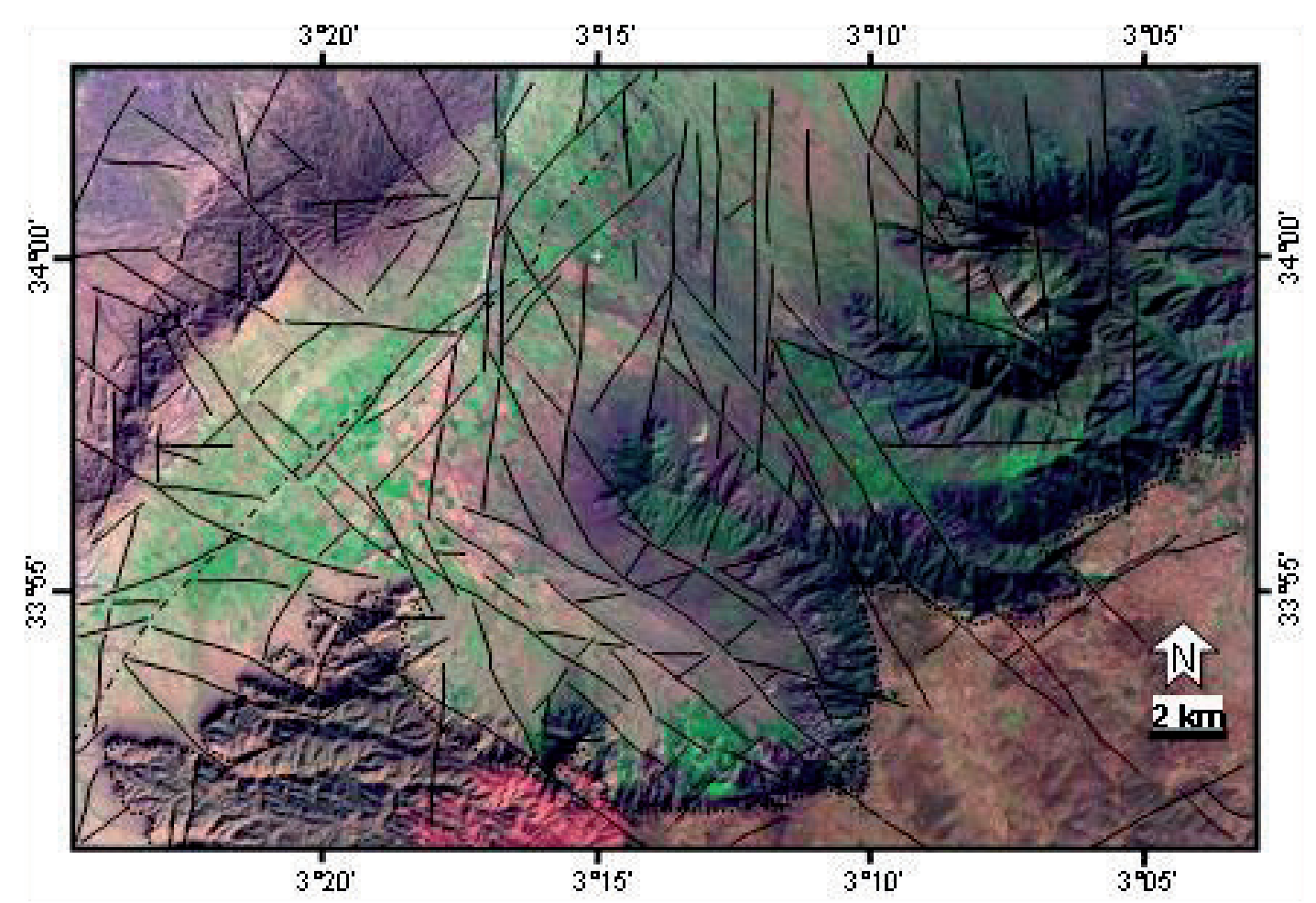

Figure 2. Composition colorée en RVB des bandes 7, 4 et 2 de Landsat ETM+, centrée sur la plaine de Tafrata et interprétation Landsat ETM+ composite color RGB (bands 7, 4 and 2) covering the Tafrata plain and interpretation. 
marquent l'influence de l'orogenèse alpine. Elles recoupent les directions précédentes et sont accompagnées sur le terrain de zones de broyage. Elles sont généralement à jeu décrochant attesté par l'existence de structures en crochon et par le décalage de talwegs.

- Les failles de direction E-O sont généralement à rejet apparent normal. Ces directions ont guidé l'évolution tectono-sédimentaire mésozoïque à l'actuelle des Hauts Plateaux septentrionaux (EL HAMMICHI et al., 2006). Les travaux de COLETTA (1977) montrent que ces failles ont été réactivées à la fin du Miocène en décrochement dextre et ont guidé la formation d'anticlinaux d'accompagnement orientés NE-SO.

\subsection{Analyse et interprétation des images radar ERS-1-SAR}

L'imagerie radar utilisée dans le présent travail correspond à la bande $\mathrm{C}$ d'acquisition (fréquence: 5,3 Ghz). Celle-ci a été obtenue en orbite ascendante. La visée se fait vers l'est avec un angle d'incidence de $23^{\circ}$ et une polarité V-V. La résolution au sol est en moyenne de $25 \mathrm{~m}$. L'image radar a fait l'objet de traitements d'amélioration radiométrique (élimination ou atténuation du chatoiement radar), test de différents filtres et de rectification géométrique. L’analyse structurale est faite par analyse visuelle à partir de documents papier restitués en noir et blanc à l'échelle de 1/250 000, de zooms donnant des agrandissements allant jusqu'à $1 / 50000$ et par visualisation et traitements sur station HP. Cette lecture répétée dans le temps, sur documents multiscalaires, est faite afin de limiter les phénomènes d'accommodation aux images et par la suite de ne représenter que les structures les plus marquées.

La carte télé-analytique obtenue (Figure 3) a été calée par confrontation aux documents topographiques et suivie d'un contrôle de terrain. Les données ont été géocodées et insérées dans des bases de données géoréférencées. Cette étude a permis d'affiner la carte de fracturation finale (Figure 4) et de définir la géométrie de la déformation.

\subsubsection{Carte de fracturation}

L'analyse de la fenêtre centrée sur la plaine de Tafrata (Figure 3) montre la présence d'unités morphologiques dont les réponses radar sont différentes. Les principaux reliefs détectés sont soulignés par la morphologie et par l'intensité de leur signal rétro-diffusé. La partie nord occidentale des Hauts-Plateaux montre un relief exaucé, relativement massif et à surface rugueuse, correspondant sur le terrain aux dépôts paléozoïques (grès, schistes) et mésozoïques (argiles, calcaires et dolomies). La plaine de Tafrata montre une forte rugosité-image où se repère un dessin régulier de bandes claires et sombres. Il définit un faciès correspondant à une différenciation lithologique des séries continentales néogènes et quaternaires.

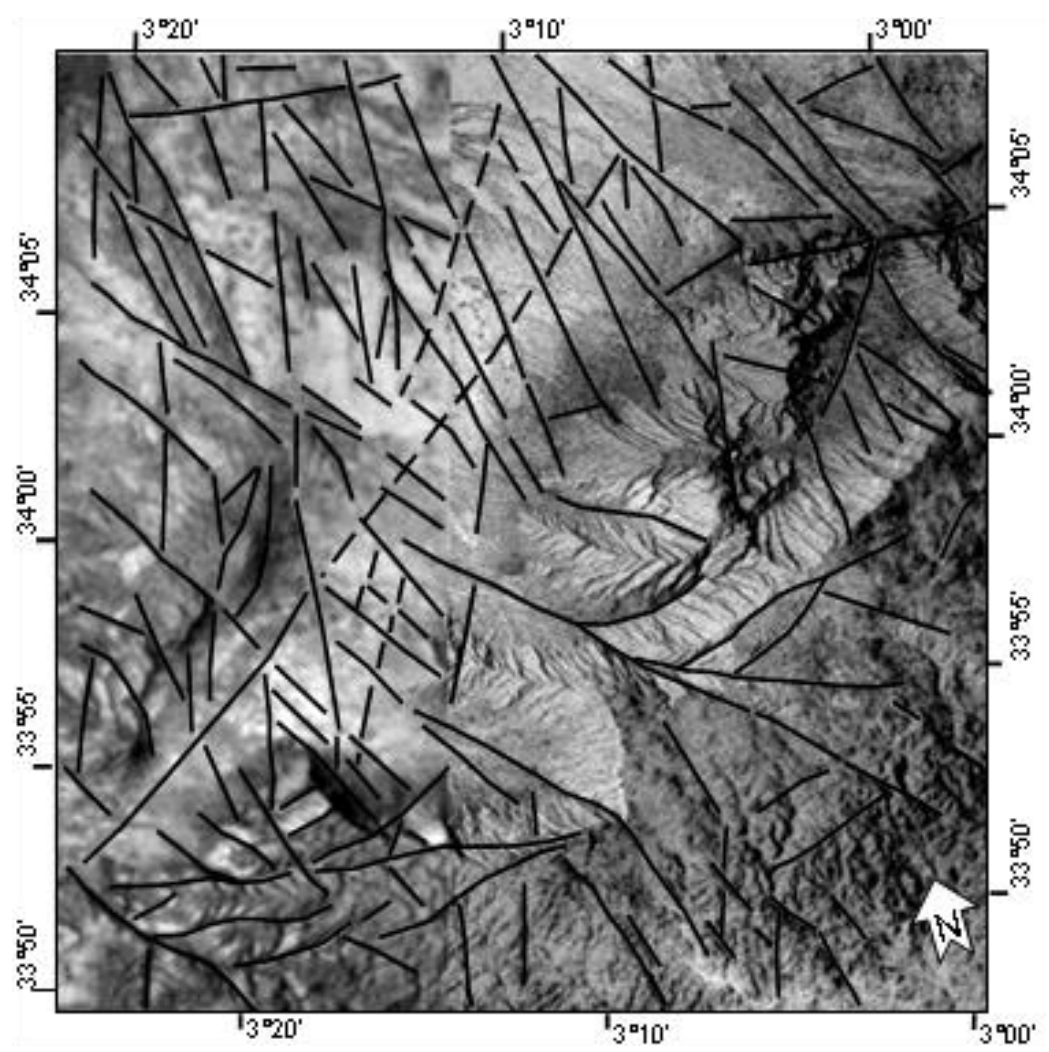

Figure 3. Image radar SAR ERS-1 centrée sur la plaine de Tafrata et interprétation. L'illumination est du SO vers le NE. Synthetic Aperture Radar (SAR) image of the first European Remote Sensing satellite (ERS-1) covering the Tafrata plain and interpretation. Illumination is from the southwest and the scene is displayed as a positive print. 


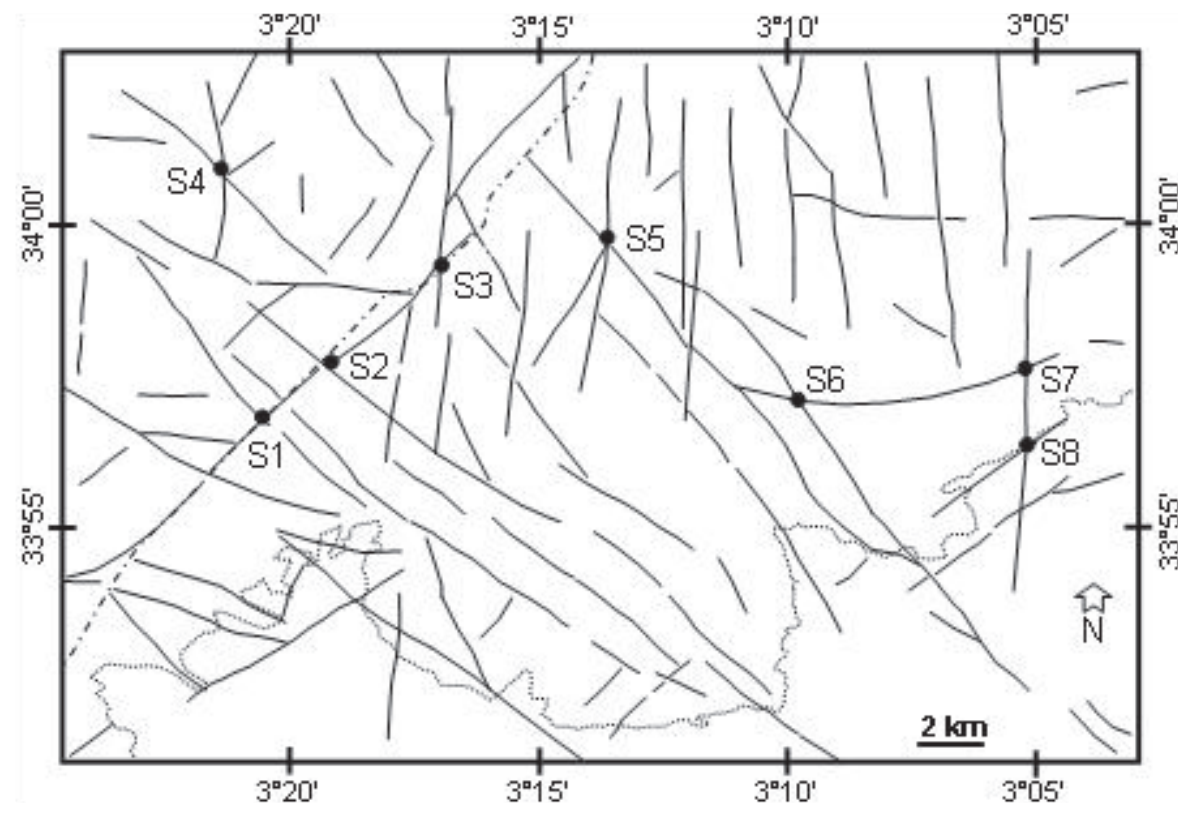

Figure 4. Carte synthétique de la fracturation centrée sur la plaine de Tafrata. Synthetic faults map of the Tafrata plain.

Dans cette zone, la pénétration des ondes électromagnétiques dans la faible couche de la couverture quaternaire, constituée de limons et conglomérats, permet de mettre en évidence un enchaînement de petites collines fortement disséquées par le réseau hydrographique. La carte de fracturation montre les mêmes directions que celles mises en évidence sur l'imagerie Landsat ETM+ (Figure 2). Il s'agit de:

- les failles de direction NE-SO montrent un tracé irrégulier sur l'image SAR-ERS-1. Ces directions s'observent au milieu de reliefs. Elles correspondent à de grands accidents verticaux à regard ouest et montrent une signature renforcée par l'intensité particulière du signal rétro-diffusé associé à leur tracé morphologique. Dans le bassin de Tafrata, ces failles sont généralement empreintées par le drainage;

- les directions NO-SE et N-S guident en surface le réseau hydrographique;

- les failles de direction E-O sont également présentes, mais difficilement reconnaissables sur l'image radar. Leur tracé paraît irrégulier suite à l'angle et la direction de la visée radar.

\subsection{Carte synthétique de la fracturation}

L’ensemble des alignements mis en évidence par télédétection ont été contrôlés sur le terrain et intégrés dans un système d'information géographique. La carte téléanalytique synthétique de la fracturation (Figure 4) montre trois directions majeures: NE-SO, NO-SE et NS. Par ailleurs, une série de petites failles de direction $\mathrm{EO}$ ne sont pas indiquées afin de ne pas surcharger la figure; elles se trouvent entre les failles les plus importantes.

\section{PROSPECTION GÉOPHYSIQUE}

Plusieurs cibles ont été choisies au croisement de linéaments hydrogéologiques importants (Figures 4 et 5). Elles ont fait l'objet d'une prospection géophysique par traînées électriques pour le positionnement exact du passage du linéament et de la résonance magnétique des protons (RMP), pour la vérification de la présence d'eau en quantité importante. Sur les huit points prospectés (Figure 4), tous ont présenté des anomalies conductrices correspondant au passage de linéaments hydrogéologiques. Cependant, seule la cible S1 a fourni un fort signal SRM correspondant à la présence d'eau en grande quantité en profondeur. Pour les besoins de cette note, un intérêt particulier sera porté pour détailler les résultats de cette cible.

\subsection{Interprétation des données de la cible S1}

En détail, cette cible fait partie d'une zone présentant l'aspect d'une pointe de flèche orientée ENE-OSO et occupant un espace d'environ $6 \mathrm{~km}$ de long sur $2 \mathrm{~km}$ dans sa plus grande largeur. Topographiquement, il s'agit d'un gradin compris entre les Hauts-Plateaux du Rekkam et la plaine de Tafrata. Le relief de la zone est constitué par une série de petites collines parallèles orientées ENE-OSO et dissymétriques. Elles montrent des flancs très raides ennoyés dans les mêmes dépôts d'alluvions et de colluvions que ceux de la plaine de Tafrata (Figure 5).

Ces collines ont le même niveau de base que celui de la plaine à laquelle elles sont reliées par une série de glacis formant un vaste plan incliné et incisé par un réseau hydrographique. 


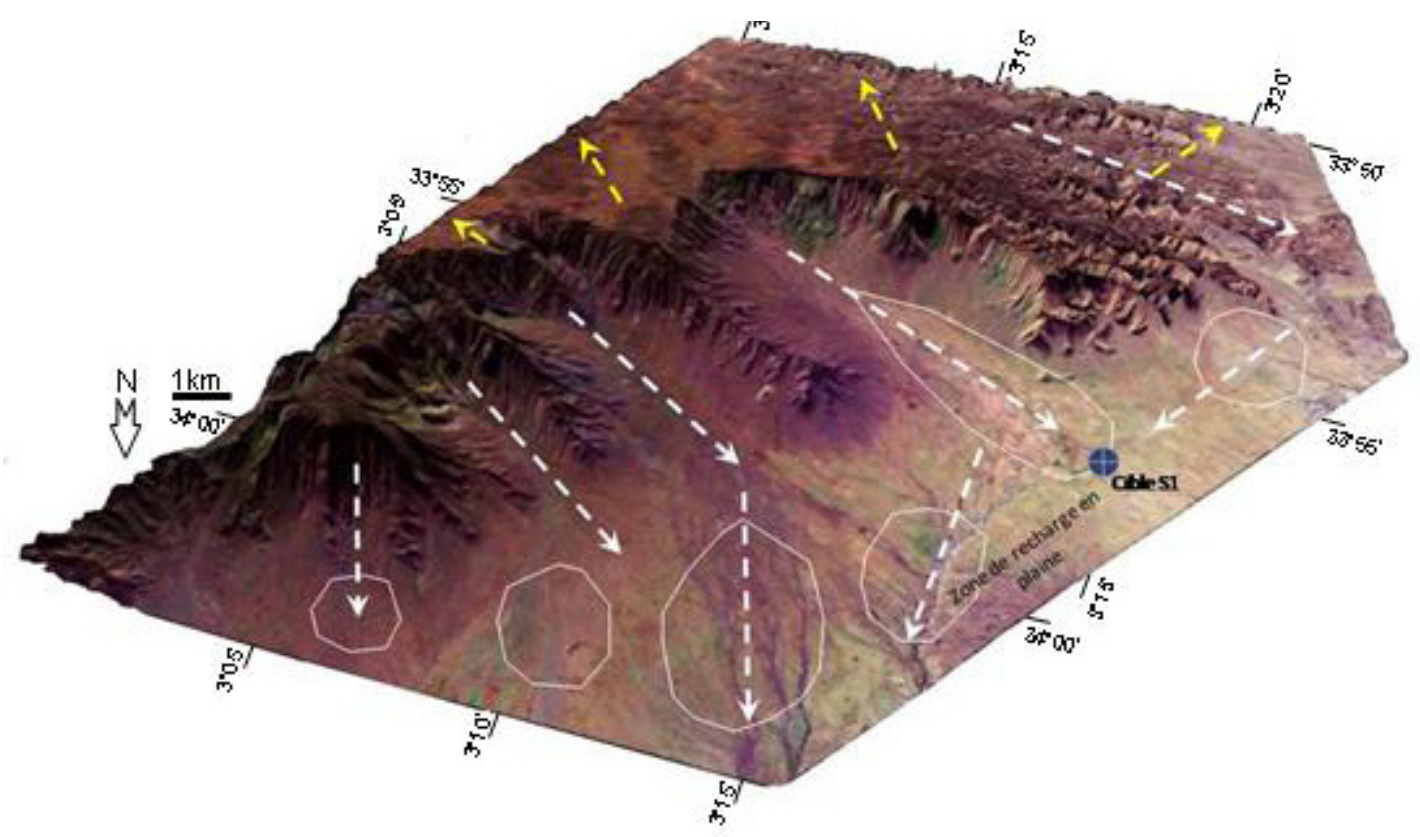

Figure 5. Modèle numérique de terrain montrant les directions d'écoulement et d'accumulation des eaux. Les flèches jaunes indiquent les directions et sens de pendages des formations géologiques et les flèches blanches correspondent aux axes de ruissellement efficace. Digital model showing the directions of flow and collection of water. The yellow arrows indicate the directions and dips of the geological formations and the white arrows correspond to the effective axis of stream.

Ce dernier témoigne d'une intense érosion linéaire en période de crues. Au plan hydrogéologique, les systèmes d'écoulement souterrains du complexe collinéen dépendent de la juxtaposition des critères suivants:

- la pente: les points hauts topographiquement sont situés à l'est mais aussi au sud;

- le pendage: il est en général vers l'ouest et localement très fort;

- la structure: la zone est bordée à l'est par un accident qui lie verticalement la partie ennoyée dans la plaine, et à l'ouest par une flexure;

- la capacité aquifere: les collines sont constituées de calcaires de nature dolomitique. La partie ennoyée est donc protégée de l'érosion aérolaire.

La recherche de cibles potentielles dans de telles conditions doit concilier les critères de 1) bas de pente; 2) situation en aval des directions de pendage; et 3) position préférable sur un bloc élevé et bien drainé. La cible $S 1$ semble rassembler l'ensemble de ces critères en plus de sa position dans une zone de convergence des linéaments hydrogéologiques (Figures 4 et 5).

\subsection{Prospection électrique}

Un sondage électrique vertical a été réalisé à l’aplomb de la cible $S 1$ en utilisant un résistivimètre de type GRM 3000 GEOSTUDI. Ce sondage a permis de déterminer l'épaisseur des différentes formations géologiques en profondeur. L'interprétation par optimisation du modèle introduit à cinq couches du sondage, réalisé par le Logiciel de traitement des données GEOSTUDI de J.L. ASTIER, montre la succession des couches géoélectriques suivantes (Figure 6 et Tableau 1):

- un dépôt résistant superficiel (Rm) (2m) qui correspond aux dépôts colluvionaires;

- au-dessous, un ensemble conducteur (C1) (9m) correspondant aux argiles rouges;

- un second dépôt résistant (R1a) dont le toit se situerait à une profondeur de $21 \mathrm{~m}$, qui pourrait correspondre à des formations marno-calcaires;

- un niveau plus résistant (R1b) correspondant vraisemblablement aux calcaires sains du Dogger ;

- et finalement un conducteur (C2) qui pourrait correspondre aux calcaires fracturés recherchés.

L'identification géologique des différentes couches s'est basée sur les observations de terrain. Les résultats du traitement mathématique de ce sondage ont permis de préciser les longueurs fixes $(200 \mathrm{~m})$ des lignes d'émission du courant utilisées pour les traînées électriques. L'utilisation de ces dernières, par un balayage latéral, permet de localiser avec précision la position des fractures: failles et contacts anormaux. Les traînées à répétition avec une double longueur de ligne permettent d'estimer le pendage de ces anomalies en profondeur.

Une traînée électrique avec une longueur d'émission AB $(200 \mathrm{~m})$ et des MN (20 m) est réalisée à l'aplomb de la cible $S 1$, selon une direction orthogonale au sein du linéament, permettant de couvrir la totalité de la zone où elle passe. Elle présente une longueur totale de $610 \mathrm{~m}$ avec une direction de déroulage E-O. Cette trainée (Figure 7) montre une anomalie 


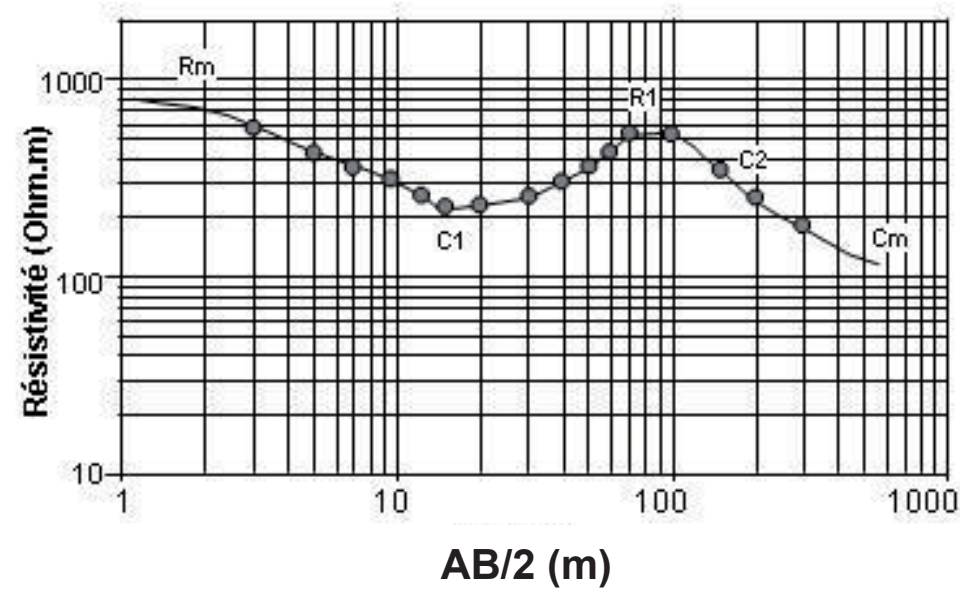

Figure 6. Sondage électrique au droit de la cible $S 1$.

Vertical electric sounding along the S1target.

Tableau 1. Résultats du sondage électrique S1.

Table 1. Results of the S1 electric survey.

\begin{tabular}{ccccc}
\hline $\begin{array}{c}\text { Couche } \\
\mathrm{n}^{\circ}\end{array}$ & $\begin{array}{c}\text { Résistivité } \\
(\text { ohm.m })\end{array}$ & $\begin{array}{c}\text { Épaisseur } \\
(\mathrm{m})\end{array}$ & $\begin{array}{c}\text { Prof. Toit } \\
(\mathrm{m})\end{array}$ & $\begin{array}{c}\text { Cote de toit } \\
(\mathrm{m})\end{array}$ \\
\hline $\mathrm{Rm}: 1$ & 800 & 1,60 & 0,00 & 716,63 \\
$\mathrm{C} 1: 2$ & 333 & 4,90 & $-1,60$ & 715,03 \\
$\mathrm{C} 1: 3$ & 107 & 4,30 & $-6,50$ & 710,13 \\
$\mathrm{R} 1: 4$ & 337 & 10,30 & $-10,80$ & 705,83 \\
$\mathrm{R} 1: 5$ & 1230 & 27,70 & $-21,10$ & 695,53 \\
$\mathrm{C} 2: 6$ & 100 & Inf & $-48,80$ & 667,83 \\
\hline
\end{tabular}

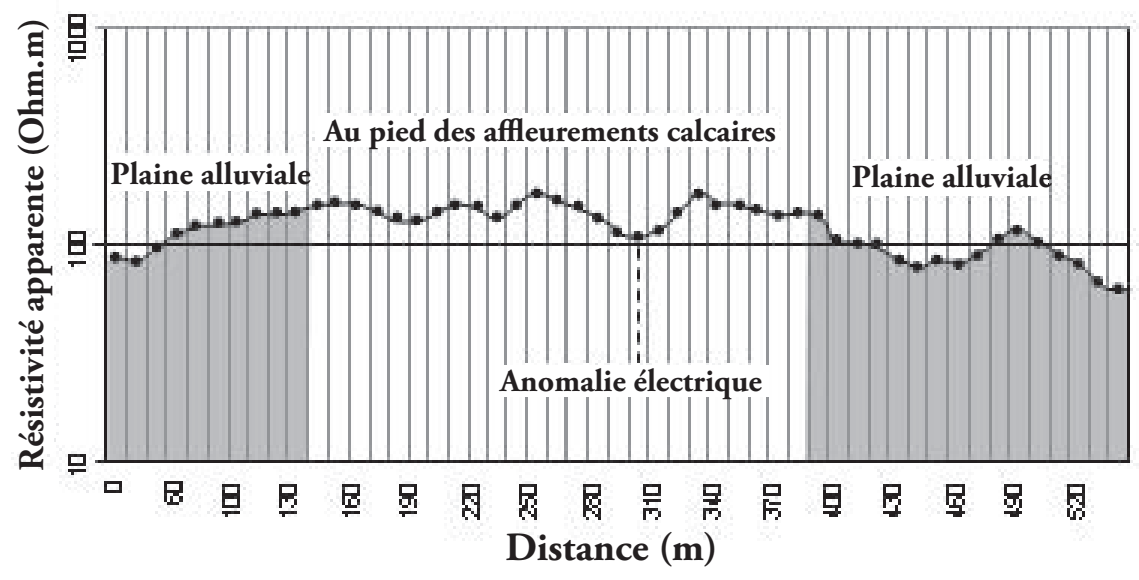

Figure 7. Traînée électrique réalisée au droit de la cible S1. Electric resistivity profiling along the S1 target. 
conductrice bien marquée au niveau de la station S17-18 avec des résistivités qui chutent depuis des valeurs de $170 \mathrm{Ohm} . \mathrm{m}$ à la station S15 à $107 \mathrm{Ohm}$.m au droit de l'anomalie. Pour tester l'aptitude du drainage d'eau en profondeur par cette faille, un sondage SRM a été réalisé au niveau de cette anomalie.

\subsection{Résonance magnétique des protons (SRM)}

La résonance magnétique des protons est une méthode de détection directe de l'eau à partir de mesures effectuées en surface (YARAMANCI et al., 2002). Son principe repose sur l'analyse du signal de résonance des noyaux d'hydrogène (ou protons) contenus dans les molécules d'eau, en réponse à un signal électromagnétique de fréquence donnée (LEGCHENKO et al., 1997; LEGCHENKO et VALLA, 2002; SCHIROV et al., 1991; VOUILLAMOZ et al., 2003). Le signal d'impulsion est créé par la circulation, dans un câble disposé en boucle au sol, d'un courant très puissant pouvant atteindre plusieurs centaines d'ampères et produit par une tension électrique de plusieurs milliers de volts (BEAUCE et al., 1996). Il crée un champ magnétique qui modifie l'équilibre énergétique des protons des molécules d'eau présentes dans le sous-sol. La coupure du courant au bout d'un temps court (quelques dizaines de millisecondes) provoque un retour à l'équilibre des protons qui renvoient alors un signal de relaxation sous la forme d'un champ électromagnétique (LEGCHENKO et al., 2002). Celui-ci, souvent faible, correspond à une différence de potentiel mesurable, de l'ordre de quelques nanovolts. Son amplitude est directement proportionnelle à la quantité d'eau présente dans le sol (LEGCHENKO et al., 2004; LUBCZYNSKI et ROY, 2003, 2004).

Le temps mis par le signal SRM pour disparaître (temps de décroissance T2*) fournit des indications sur l'environnement direct des nappes: il est d'autant plus long que les protons sollicités sont ceux d'une eau peu enserrée dans la roche, donc d'une nappe à potentiel hydrodynamique élevé. Les mesures du courant SRM sont sensibles au bruit électromagnétique (lignes de haute tension, antennes radios, pompes hydrauliques, etc.) et sont gênées par la présence de roches magnétiques. Dans notre cas, 6/8 des anomalies conductrices ont pu faire l'objet de sondages SRM, puisque sur les deux anomalies restantes (S2 et S3), l'importance de l'amplitude du bruit ambiant rendait les sondages inexécutables. Les sondages SRM ont été exécutés à l'aide du matériel NUMIS Plus (IRIS INSTRUMENT) avec une boucle de $75 \times 75 \mathrm{~m}$. Il faut cependant mentionner que des mesures du champ magnétique local, à l'intérieur de la boucle, ont précédé l'opération d'acquisition des données, pour relever toute influence de la variation de champ magnétique pouvant être causée par des roches magnétiques, des lignes électriques, etc. Les valeurs du champ magnétique, réalisées par un magnétomètre standard, sont restées relativement constantes (entre 41703 et 41715 nT).
Au cours de l'acquisition des données des sondages SRM par le logiciel «Prodiviner NUMIS Acquisition » et après inversion mathématique à l'aide du logiciel «Samovar Program», un seul sondage ( $\mathrm{S} 1)$ a présenté un bon signal SRM, observé entre les valeurs 5300 et 10000 (A-ms) des moments d'impulsion, avec une amplitude maximale de l'ordre de $124 \mathrm{nV}$ (Figure 8). Sur la totalité des autres sondages exécutés dans cette étude, aucun n’a dépassé les $45 \mathrm{nV}$, comme en témoignent les quelques sondages représentés dans la figure 8 .

La courbe de variation de l'amplitude du signal du SRM S1 montre une forme en cloche avec une augmentation progressive entre 2000 et 3600 (A-ms) des valeurs enregistrées des moments d'impulsion. Ils indiquent vraisemblablement l'humidité rencontrée à l'approche de l'eau (Figure 8). Le signal SRM, correspondant à la présence d'eau, est enregistré entre les valeurs 5300 et 10000 (A-ms) des moments d'impulsion.

Les fréquences répétitives $(1776 \mathrm{~Hz})$ enregistrées entre les valeurs 3600 et 10000 (A-ms) des moments d'impulsion, correspondant à la fréquence Larmor, calculée à partir du champ magnétique local, montrent que ce signal correspond à une présence d'eau en profondeur. Les valeurs positives et rapprochées de la phase (Figure 9) confirment ce résultat.

La constante de temps $\mathrm{T} 2 *$ enregistre des valeurs importantes (400-450 ms) aux alentours des valeurs 5300 et 10000 (A-ms) des moments d'impulsion, ce qui indique des conditions hydrodynamiques favorables et une perméabilité de fissure importante dans cette tranche de profondeur (Figure 10). Nous signalons que les conditions d'acquisition du sondage sont idéales puisque les valeurs enregistrées du bruit ambiant, avec sommation, pouvant altérer l'enregistrement du signal d'eau, sont relativement faibles (inférieures à $70 \mathrm{nV}$ ) (Figure 8).

L'inversion mathématique du sondage SRM, en utilisant la matrice calculée à partir du sondage électrique vertical réalisé, montre une tranche d'eau correspondant au signal, dont le toit est situé à $44 \mathrm{~m}$ de profondeur (Figure 11). Le volume d'eau calculé, par unité surfacique, correspondant à ce signal est de $\mathrm{V}(\mathrm{eau})=3,2 \mathrm{~m}^{2} \cdot \mathrm{m}^{3}$. Ce sondage a fait l'objet d'un forage mécanique de $80 \mathrm{~m}$ de profondeur, dont le débit après essais de pompage a atteint $144 \mathrm{~m}^{3} \cdot \mathrm{h}^{-1}$. Les résultats de ce forage corroborent ceux du SRM puisque la venue d'eau a été observée à $42 \mathrm{~m}$ de profondeur.

Cette étude montre l'importance de l'utilisation de la résonance magnétique des protons pour l'optimisation du choix des cibles pouvant faire l'objet de futurs forages positifs. Cependant, l'existence d'une seule cible, présentant un potentiel d'eau important, laisse penser que celle-ci est située dans une zone de recharge du linéament qui est très favorable. 

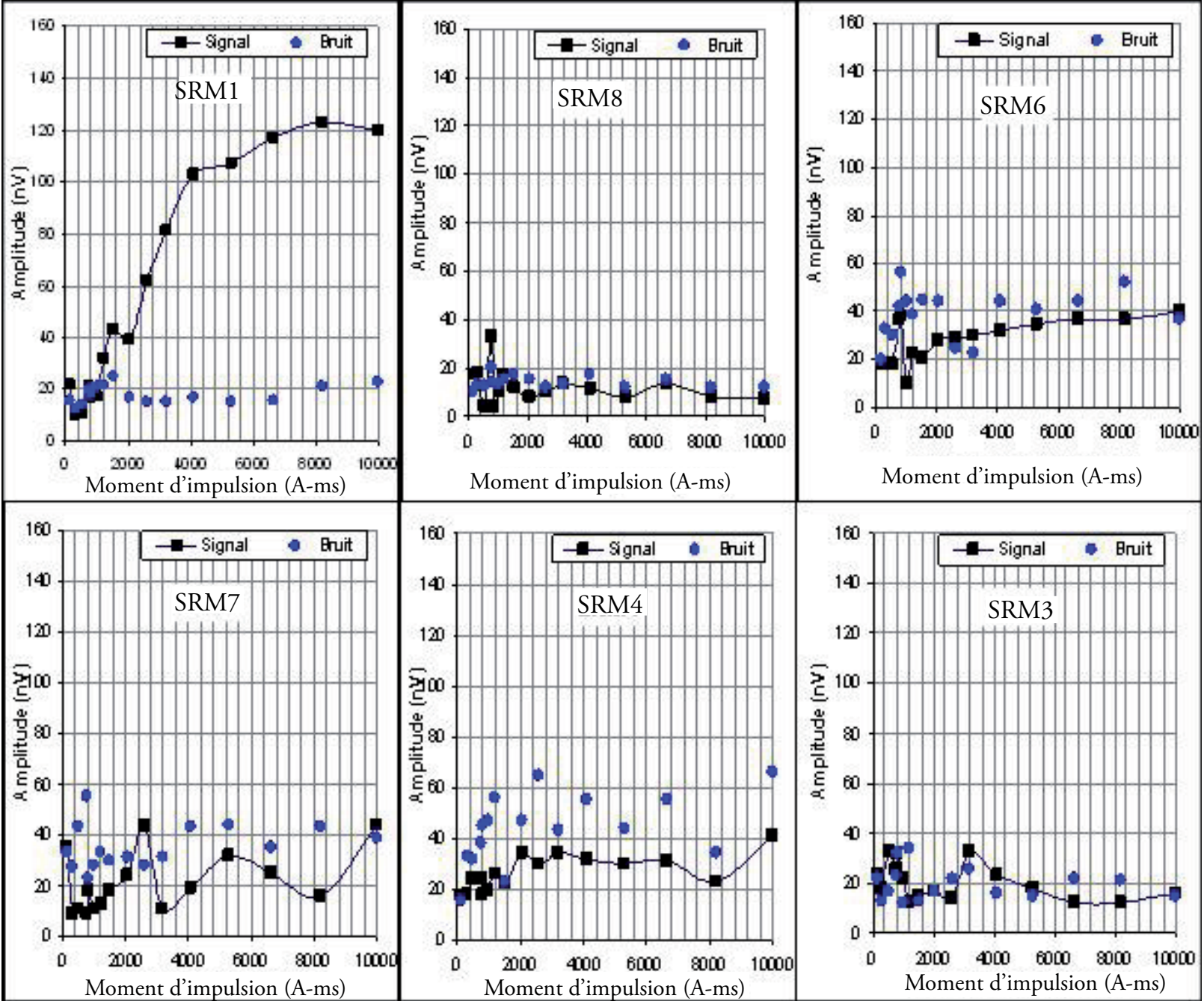

Figure 8. Variation de l'amplitude du signal et du bruit de quelques sondages SRM au cours des impulsions. Variation of amplitude of the signal and noise of some MRP drillings during the impulses.

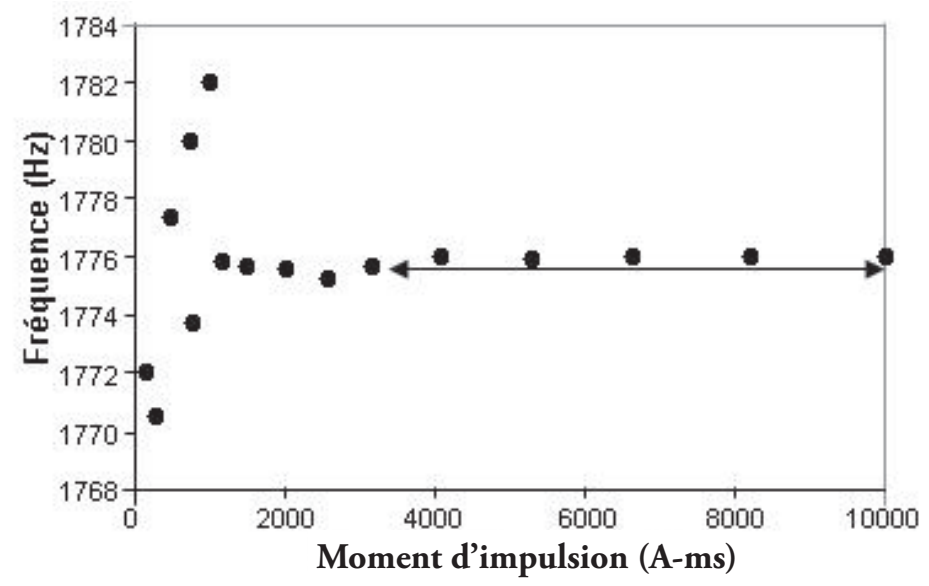

Figure 9. Variation de la fréquence lors de l'acquisition du sondage SRM S1.

Variation of the frequency during the acquisition of the MRP drilling S1.

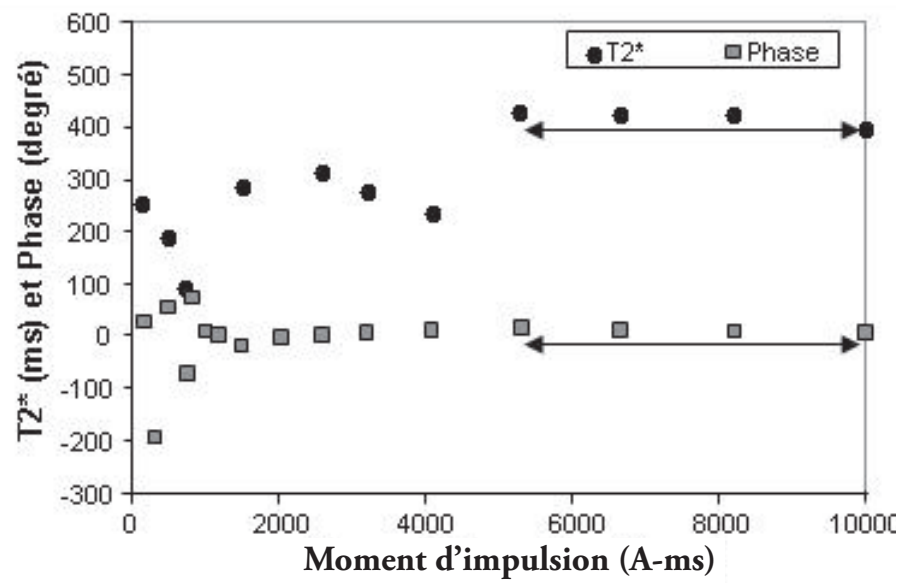

Figure 10. Variation de la constante de temps $\mathrm{T} 2^{*}$ et de la phase lors de l'acquisition du sondage SRM S1. Variation of the time-constant $T 2^{*}$ and the phase during the acquisition of the MRP drilling S1. 


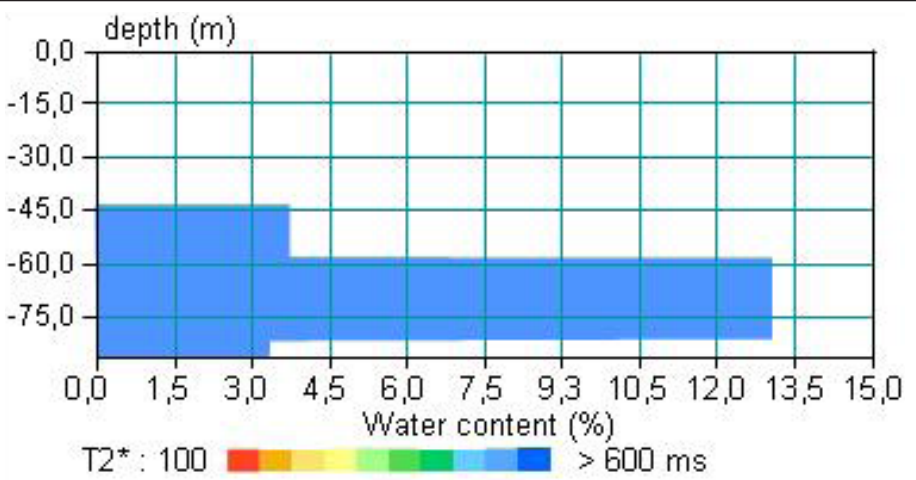

Figure 11. Résultats de l'inversion mathématique du sondage SRM S1.

Results of the mathematical inversion of the MRP drilling $S 1$.

\section{RÉSULTATS ET DISCUSSION}

Les résultats de cette étude montrent que la zone de recharge de la cible $S 1$ ne peut pas correspondre aux eaux en provenance de la plaine de Tafrata puisque celle-ci se trouve sur un compartiment effondré. Elle ne peut non plus provenir de la partie orientale de la plaine puisque le socle paléozoïque affleure et forme un écran imperméable pour l'infiltration des eaux. Les quantités d'eaux ruisselées et infiltrées sur la retombée $\mathrm{du}$ plateau sont trop faibles pour fournir une alimentation compatible avec les volumes issus des essais de pompage réalisés au cours du forage $S 1$.

La seule origine viable est un drainage souterrain par un accident bordier ENE-OSO, déjà déterminé par le traitement de l'image satellitale. Ce collecteur constitue un drain pour les eaux des oueds descendants des Hauts-Plateaux sur une zone tampon d'une vingtaine de $\mathrm{km}$ du sud vers le nord. L'un des critères les plus remarquables ressortant de l'interprétation des données satellitaires est la bonne recharge qui existe en périphérie des plateaux. La zone de ruissellement et d'infiltration de l'amont est de l'ordre de $400 \mathrm{~km}^{2}$. Elle est très peu végétalisée et ne présente pas d'utilisation agricole. Le potentiel drainé vers les aquiferes est donc actuellement intact. Par ailleurs, du fait de la présence d'un minimum annuel de pluie tombée $(350 \mathrm{~mm})$, on estime qu'à la profondeur explorée $(100 \mathrm{~m})$, on se situe bien dans une zone de recharge annuelle et non pas dans un aquifère fossile. En absence de budgets alloués à la réalisation d'études similaires dans cette zone, la meilleure stratégie d'implantation de futurs points d'eau est la proximité des accidents bordiers du plateau calcaire situés dans les compartiments formant les horsts. C'est ainsi que l'on a le plus de chances d'atteindre une cible répondant aux exigences rapides et urgentes de différentes localités.

\section{CONCLUSION}

Une méthode multicritère pour l'optimisation des sites d'implantation des forages a été appliquée dans la plaine de Tafrata en contexte semi-aride (Maroc nord-oriental). Le traitement et l'interprétation de l'imagerie satellitale a permis de cartographier l'essentiel des linéaments hydrogéologiques présents au niveau de cette région. La mise en évidence d'anomalies conductrices au niveau des trainées électriques, réalisée au droit des points de jonction des linéaments, permet de les localiser. L'inversion mathématique du sondage à résonance magnétique des protons, réalisée au droit de l'anomalie électrique $\mathrm{S} 1$, montre un important signal correspondant à la présence d'eau en profondeur. Elle indique la présence d'une tranche d'eau dont le toit se situerait à $44 \mathrm{~m}$ de profondeur. Le sondage mécanique réalisé confirme ces résultats, puisque la venue d'eau a été rencontrée à $42 \mathrm{~m}$ au niveau des deux horizons cités ci-dessus. Cette méthodologie a prouvé sa fiabilité d'utilisation dans les régions hydrogéologiques discontinues à déficit hydrique.

\section{BIBLIOGRAPHIE}

AIT BRAHIM L. (1986). La zone de fracture trans-marocaine d'Agadir-Nékor: critères géophysiques, données de terrain et analyse de documents Landsat. Bull. Inst. Scient., Rabat, $10,27-40$.

AIT BRAHIM L., H. TABYAOUI, A. ABDELOUAFI, et P. CHOTIN (2000). Modèle de déformation de l'avantpays rifain (Monts des Béni Snassen, Maroc) au Mio-pliocène à partir des données microtectoniques et satellitales (Spot, ERS1). Photo-Interprétation, ESKA (Éditeur),Paris, France. 2000/1-2, pp. 21-58.

BEAUCE A., J. BERNARD, A. LEGCHENKO et P. VALLA (1996). Une nouvelle méthode géophysique pour les études hydrogéologiques: l'application de la résonance magnétique nucléaire. Hydrogeology 1, 71-77.

BENZAQUEN M. (1965) Étude stratigraphique préliminaire des formations du bassin de Guercif. Rapport inédit, Serv. Géol. Maroc, No 30853, 74 p., 11 figures.

BERNINI M., M. BOCCALETTI, J. EL MOKHTARI, R. GELATI, G. MORATTI et G. PAPANI (1994). Geologic-structural map of the Taza-Guercif Neogene basin (North Eastern Morocco). Scale 1:50 000. S.EL.CA (Éditeur), Firenze, Italie. 
CHOTIN P., L. AIT BRAHIM, B. DEFFONTAINES, J.P. RUDANT et A. CHAOUNI (1995). Apports des données ERS-1 SAR sur la reconnaissance du réseau de failles dans la péninsule de Tanger (Maroc). Photo-Interprétation, 2, 146-152.

COLETTA B. (1977). Évolution néotectonique de la partie méridionale du bassin de Guercif (Maroc oriental). Thèse du $3^{\text {e }}$ Cycle, Univ. Grenoble, France, 165 p.

CONDID C.D. et P.S. CHAVEZ (1979). Basic concepts of computerized digital image processing for geologists. U.S. Geol. Surv. Bull., 1462, 16 p.

DESJARDINS R.S., D.W. IRIS, G.H. ROY, G. LEMIEUX et T. TOUTIN (2000). Efficacité des données de Radarsat-1 dans la reconnaissance des linéaments: un bilan. J. Can. Télédétection, 26, 537-548.

EL HAMMICHI F., H. TABYAOUI, A. CHAOUNI, L. AIT BRAHIM et P. CHOTIN (2006). Mio-Pliocene tectonics in Moroccan rifian foreland: coexistence of compressive and extensional structures. Revta Soc. Geol. Es., 19, 143-152.

EMRAN A., J. CHOROWICZ, B. CERVELLE, N. LYBERIS, G. TAMAIN et E.M. ALEM (1988). Cartographie géologique et analyse de la fracturation du sud de l'Anti-Atlas central (Maroc) à partir d'une image Landsat MSS, Photo Interprétation, 2, 1-7.

HIMYARI M.S., C. HOEPFFNER, M. BENZAKOUR et D. EL HADANI (2002). Étude structurale du Haut-Atlas oriental (Maroc) à l'aide de l'analyse linéamentaire des images HRV (XS) de SPOT. Télédétection, 2, 243-253.

HOBBS W.H. (1904). Lineaments of the Atlantic border region. Geol. Soc. Amer. Bull., 15, 483-506.

ISRAEL G., M. GOLDMAN, B. RABINOVICH, M. RABINOVICH et A. ISSAR (1996). Detection of the water level in fractured phreatic aquifers using nuclear magnetic resonance (NMR) geophysical measurements. J. Applied Geophys., 34, 277-282.

LEGCHENKO A.V., J.M. BALTASSAT, A. BEAUCE et D. CHIGOT (1997). Application of proton magnetic resonance for detection of fractured chalk aquifers from the surface. Dans : Proceedings of the $3^{\text {rd }}$ Meeting of the Environment and Engineering Geophysics (EEGS), EEGS Aarhus'97 Meeting, Danemark, 8-11 septembre 1997.
LEGCHENKO A. et P. VALLA (2002). A review of the basic principles for proton magnetic resonance sounding measurements. J. Appl. Geophys., 50, 3-19.

LEGCHENKO A.V., J.M. BALTASSAT, A. BEAUCE et J. BERNARD (2002). Nuclear magnetic resonance as a geophysical tool for hydrogeologists. J. Appl. Geophys. 50, 21-46.

LEGCHENKO A., J.M. BALTASSAT, A. BOBACHEV, C. MARTIN, H. ROBAIN et J.M. VOUILLAMOZ (2004). Magnetic resonance sounding applied to aquifer characterization, J. Ground Water, 42, 363-373.

LUBCZYNSKI M.W. et J. ROY (2003). Hydrogeological interpretation and potential of the new Magnetic Resonance Sounding (MRS) method. J. Hydrol., 283, 19-40.

LUBCZYNSKI M.W. et J. ROY (2004). Magnetic resonance sounding (MRS) - new method for ground water assessment. J. Ground Water, 2, 291-304.

MAHMOUD A. (1996). Lineament as groundwater exploitation guides in hard-rock terranes of arid region, J. Can. Télédétection, 22, 108-116.

O'LEARY D.W., J.D. FRIEDMAN et H.A. POHN (1976). Lineament, linear lineation some proposed new standards for old terms. Geol. Soc. Am. Bull., 87, 1463-1469.

ROUZEAU O. et J. SCANVIC (1989). Interprétation structurale des linéaments observés sur des données spatiales thermiques multidate et multiplicateur dans le bassin de Paris. Bull. B.R.G.M, Orléans, France, 113, 8-12.

SCHIROV M., A. LEGCHENKO et G. CRÉER (1991). A new direct non-invasive groundwater detection technology for Australia. Explor. Geophys., 22, 333-338.

SHARMA K.N.M., V.H. SINGHROY, L. MADORE, J. LÉVESQUE, C. HÉBERT et M. HINSE (1999). Use of radar images in the identification of major regional structures in the Greenville province, western Quebec, Can. J. Remote Sens., 25, 278-290.

SIEGAL B.S. et M.J. ABRAMS (1976). Geological mapping using Landsat data. Photogramm. Eng. Remote Sens., 42, 325-337.

VOUILLAMOZ J.-M., A. LEGCHENKO, Y. ALBOUY, M. BAKALOWICZ, J.M. BALTASSAT et W. ALFARES (2003). Localization of saturated karst aquifer with magneticresonance sounding and resistivity imagery. $J$. Ground Water, 41, 578-587. 
WERNLI R. (1988). Micropaléontologie du Néogène post-nappes du Maroc septentrional et description systématique des foraminiferes planctoniques. Notes et Mem. Serv. Géol. Maroc, 331, 270 p.

YARAMANCI U., G. LANGE et M.HERTRICH (2002). Aquifer characterization using Surface NMR jointly with other geophysical techniques at the Nauen/Berlin test site. J. Appl. Geophys., 50, 47-65.

ZIZI M. (1996). Triassic-Jurassic extension and Alpine inversion in the northern Morocco. Mem. Mus. Natl. His. Nat., 170, 87-101. 